\title{
Using Spatial Division Multiplexing to avoid fragmentation in Flex-Grid Optical Networks
}

\author{
Jaume Comellas, Jordi Perelló, Josep Solé-Pareta, Gabriel Junyent \\ Universitat Politècnica de Catalunya (UPC) - BarcelonaTech, Barcelona, Spain \\ E-mail: jaume.comellas@upc.edu
}

\begin{abstract}
Spectrum fragmentation is one of the main drawbacks of Flex-Grid Optical Networks, and it becomes especially harmful under dynamic traffic conditions. When evolving from single fibre networks to spatial division multiplexed (SDM) ones, where many parallel cores/fibres are deployed, spectrum fragmentation still limits their eventual performance. In this work, the total available spectrum is partitioned according to the SDM dimension, and each one of the resulting partitions is assigned to each type of connections. This strategy is proposed as a simple method to eliminate spectrum fragmentation. By allocating all same sized connections onto the same spectral/spatial partition, the randomness in the size of the spectral voids left by torn down connections is eliminated. The proposal is numerically evaluated using different network topologies. The obtained results show that spatial partitioning is an interesting solution for spectrum fragmentation avoidance in these scenarios.
\end{abstract}

Keywords: Elastic Optical Network; Spatial Division Multiplexing; fragmentation avoidance.

\section{INTRODUCTION}

Elastic Optical Networks (EON) based on the Flex-Grid concept [1] have become the right solution to develop the near future data transport networks, since they offer higher spectral efficiency than those based on Wavelength Division Multiplexing (WDM). In Flex-Grid networks, the fibre optic spectrum is divided into 12.5 $\mathrm{GHz}$ frequency slots (FS), which are contiguously assigned to create channels adapted to any signal bandwidth. This enables the efficient allocation of heterogeneous connections together onto the same optical network fibres. On the other hand, Spatial Division Multiplexing (SDM) [2] has been recently introduced as the right strategy to further increase network capacity, so as to cope with the expected traffic forecasts. In SDM networks, Single Mode Fibre Bundles (SMFBs) can be equipped per link in a near-term, thus multiplying the Flex-Grid network capacity by the number of parallel independent SMFs per bundle. The mid- and long-term fully-SDM vision is expected to rely on advanced optical fibre technologies [3], like Multi-Core Fibres and Few-Mode Fibres.

Spectrum fragmentation [1] was identified as a major issue in EONs since they were proposed. Fragmentation results from the allocation and release of spectral resources demanded by connections with different bandwidth requirements, leaving spectral gaps of different widths that difficult the allocation of future connections, particularly those with high spectral requirements. To mitigate fragmentation effects, proactive and reactive solutions have been proposed in the literature [4] [5]. Reactive solutions aim at defragmenting the optical fibre spectrum, thus enabling the potential allocation of future connection requests. On the other hand, proactive solutions allocate new connections trying to minimize the resulting fragmentation.

A proactive solution proposed in the literature to avoid the pernicious effects of the spectrum fragmentation in Flex-Grid optical networks consists in dividing the spectral resources, so that each of the resulting partitions is dedicated to only support connections with identical bandwidth requirements [6][7]. In this way, by applying a simple first-fit spectrum assignment strategy, the width of the available spectrum gaps in each partition will always match the bandwidth requirements of future connections to be allocated there. A critical aspect to achieve high spectral utilization with this solution is to assign an appropriate amount of spectrum to each partition, for example, based on expected load and traffic profile.

The present paper has a two-fold objective. First of all, we propose a proactive fragmentation avoidance solution relying on resource partitioning for Flex-Grid/SDM networks. Two different approaches are revised: exact partitioning, where the quantity of FSs assigned to each type of connections is proportional to the ratio of spectrum used by them, and spatial partitioning, where an integer number of spatial channels (fibres/cores) is assigned to each type of connections. Then, we numerically compare the performance of these solutions with a model where all the resources are shared among all the connection types. The remainder of this paper continues as follows. Section 2 presents the proposed resource partitioning solutions for Flex-Grid/SDM networks, highlighting their advantages. Section 3 presents the evaluation scenarios, while illustrative simulation results are shown in Section 4. Main conclusions are drawn up in Section 5.

\section{AVOIDING FRAGMENTATION BY SPATIAL SPECTRUM PARTITIONING}

A common characteristic of any Flex-Grid/SDM scenario is the coexistence of heterogeneous connections, such that the spectrum allocated to them fits their bandwidth requirements. Many previous works dealt with multi-rate 
connections, whose spectral needs vary depending on the modulation formats used. Considering the available technology, we will assume dynamic network scenarios simultaneously supporting $100 \mathrm{G}, 400 \mathrm{G}$ and $1 \mathrm{~Tb} / \mathrm{s}$ connections, tailoring their spectrum assigned to their needs, therefore creating spectral fragmentation.

By extending the idea provided in [6] and [7] for Flex-Grid/SMF networks to Flex-Grid/SDM ones, we propose to isolate the different connection types by assigning them different spectral resource partitions, what we refer to as spectral partitioning. According to the traffic profile offered to the network, different spectrum partitions are created, whose sizes depend on the offered ratio of each type of connections. These partitions can include spectral resources belonging to multiple spatial channels present in the Flex-Grid/SDM network links.

The offered traffic profile assumed throughout this work is composed of $100 \mathrm{G}(40 \%), 400 \mathrm{G}(30 \%)$ and $1 \mathrm{~T} / \mathrm{s}$ $(30 \%)$ connections (later on referred to as 40-30-30 profile). By simple calculation, it is found that, in average, $100 \mathrm{G}$ connections contribute to $8.7 \%$ of the total offered traffic, while $400 \mathrm{G}$ and $1 \mathrm{~Tb} / \mathrm{s}$ connections contribute to $26.1 \%$ and $65.2 \%$ of it, respectively. Indeed, for $100 \mathrm{G}$ connections, the following calculation applies: $40 \% * 100 \mathrm{G} /(0.4 * 100+0.3 * 400+0.3 * 1000)=8.7 \%$. This calculation is almost identical for $400 \mathrm{G}$ and $1 \mathrm{~Tb} / \mathrm{s}$ connection types. In general, if the expected traffic profile is known, the available spectral resources could be partitioned using these values.

Nevertheless, to be more precise, it is also interesting to take into account how the different connections are mapped onto the spectrum. This depends on the baud-rate and modulation formats used. As an example, let us assume that modulators have a fixed baud-rate of 25 Gbaud and Polarization Multiplexed (PM)-8-QAM is used (with a spectral efficiency equal to $6 \mathrm{bit} / \mathrm{s} / \mathrm{Hz}$ ). One additional FS is left for Guard-Band (GB) between adjacent Optical Carriers (OCs), so each OC requires the allocation of 3 FSs in total, with an effective transmission capacity of $150 \mathrm{~Gb} / \mathrm{s}$. Then, $100 \mathrm{G}, 400 \mathrm{G}$ and $1 \mathrm{~Tb} / \mathrm{s}$ connections would demand super-channels composed of 1 , 3 and 7 OCs, respectively, requiring 3, 9 and $21 \mathrm{FSs}$ in total. If such required spectral resources per connection type are considered instead of their contribution to the offered traffic, $100 \mathrm{G}$ connections are expected to consume the $11.75 \%$ of the available spectral resources, while $400 \mathrm{G}$ and $1 \mathrm{~Tb} / \mathrm{s}$ the $26.5 \%$ and $61.75 \%$, respectively. These values are obtained by similar calculations as the ones before. For example, for 100G connections we find that $40 \% * 3 \mathrm{FS} /(0.4 * 3+0.3 * 9+0.3 * 21)=11.75 \%$. When a different modulation format is used, the mapping between traffic and spectrum used changes.

The available spectral resources can be divided according to such expected percentages and the resulting spectrum partitions assigned to each type of connections. For example, assuming that links have 12 spatial channels (e.g., a SMFB composed of 12 parallel SMFs or a 12-cores MCF), each one consisting of the entire 4 $\mathrm{THz}$ C-Band discretized into $320 \mathrm{FSs}$ of $12.5 \mathrm{GHz}$, a total of $3840 \mathrm{FSs}$ are available among all the spatial channels. So, considering the spectral consumption ratios per connection type presented in the previous paragraph (hereafter called SPECP strategy), the total spectral resources should be partitioned so that 451, 1018 and $2371 \mathrm{FSs}$ are dedicated to $100 \mathrm{G}, 400 \mathrm{G}$ and $1 \mathrm{~Tb} / \mathrm{s}$ connections, respectively. When a new connection of a specific type (i.e., $100 \mathrm{G}, 400 \mathrm{G}$ or $1 \mathrm{~Tb} / \mathrm{s}$ in our examples) arrives into the network, the spectrum allowed to allocate it is limited to these partitions, selected in a first-fit fashion. Two advantages are foreseen when adopting this strategy vs. generic Flex-Grid: 1) Fragmentation is avoided as spectrum bands released after connections termination have the same size inside the different partitions; 2) The unfairness problem [7] typical of Flex-Grid scenarios, where large connections suffer higher blocking than smaller ones, is avoided. Indeed, by using this mechanism partitions could be tailored to tune the network blocking performance.

An alternative to this approach, applicable to Flex-Grid/SDM network scenarios is a simpler spatial partitioning, where full spatial channels (fibres/cores) are dedicated to each traffic type (hereafter called SPAP strategy). Taking again the expected spectral resource consumption of $100 \mathrm{G}, 400 \mathrm{G}$ and $1 \mathrm{~Tb} / \mathrm{s}$ connections (i.e., $11.75,26.5$ and $61.75 \%$ ), we can easily obtain that, out of the 12 available spatial channels, $1.41,3.18$, and 7.41 have to be dedicated to them, respectively. Rounding these values, 1 spatial channel would be dedicated to support $100 \mathrm{G}$ connections, 3 of them to support $400 \mathrm{G}$ connections, and the remaining 8 to support $1 \mathrm{~Tb} / \mathrm{s}$ ones. It is obvious that in this case $100 \mathrm{G}$ and $400 \mathrm{G}$ connections are penalized, while more FS than the required are assigned to $1 \mathrm{Tbit} / \mathrm{s}$ connections. Nevertheless, this effect would be mitigated in scenarios where the number of spatial channels is increased and, therefore, the rounding effects are smaller. The main objective of this work consists in demonstrating these effects by means of extensive simulations in different network scenarios.

\section{SIMULATED SCENARIOS}

An ad-hoc Java-based simulator has been developed to obtain accurate numerical results about the performance of the previously described spectral and spatial partitioning strategies for Flex-Grid/SDM network scenarios.

Two different topologies [8] have been considered for this study, namely, the DT12 German transport network (12 nodes, 20 links), whose diameter is $1019 \mathrm{~km}$, and the Pan-European EON16 network (16 nodes, 23 links), with a diameter of $2663 \mathrm{~km}$. For the sake of simplicity, a single modulation format is employed in each network. According to [9], the maximum transmission reach of PM-8QAM is $1340 \mathrm{~km}$ in SDM networks deploying SMFB links, while this reach is increased to $3796 \mathrm{~km}$ when PM-QPSK is used. Therefore, when the DT12 is simulated, PM-8QAM is assumed and the maximum path length is limited to $1340 \mathrm{~km}$. This means that 3,9 and 21 adjacent 
FSs will be required to allocate $100 \mathrm{G}, 400 \mathrm{G}$ and $1 \mathrm{~Tb} / \mathrm{s}$ connections, respectively. In contrast, when simulating the EON16, the maximum path length increases to $3796 \mathrm{~km}$, as PM-QPSK will always be employed. Nonetheless, 3, 12 and 30 adjacent FSs will be needed to allocate $100 \mathrm{G}, 400 \mathrm{G}$ and $1 \mathrm{~Tb} / \mathrm{s}$ connections there. A SMFB is assumed to be deployed per network link in each direction, including 12 standard SMFs (the 4 THz CBand is used in each SMF, which results in $320 \mathrm{FSs}$ of $12.5 \mathrm{GHz}$ ). Regarding the Routing, Spatial channel and Spectrum Assignment (RSSA) algorithm, we employ a k-Shortest Path routing algorithm (where k=3), constraining the candidate paths by the aforementioned maximum path length values. Once the candidate paths to support a given connection are obtained, they are explored starting from the shortest to the longest one. As for the spatial channel and spectrum assignment, a first-fit strategy is used, and spatial channel continuity must be ensured end-to-end along the candidate paths, besides the spectrum continuity and contiguity constraints imposed by the Flex-Grid technology.

As for the generated traffic profile, the respective percentages of $100 \mathrm{G}, 400 \mathrm{G}$ and $1 \mathrm{Tbit} / \mathrm{s}$ connections are $40 \%, 30 \%$ and $30 \%$ (40-30-30 profile). It is important to highlight that the fraction of spectrum expected to be required per connection type differs from their contribution to the total offered traffic in the DT12 network, but not in the EON16 one. For example, $100 \mathrm{G}, 400 \mathrm{G}$ and $1 \mathrm{~Tb} / \mathrm{s}$ connections will contribute to $8.7 \%, 26.1 \%$ and $65.2 \%$ of the total offered traffic, which matches their spectrum requirements in the EON16. This is due to the fact the ratio of FSs needed (3, 12 and $30 \mathrm{FSs})$ vs. connection bit-rate $(100 \mathrm{G}, 400 \mathrm{G}, 1 \mathrm{~Tb} / \mathrm{s})$ keeps constant when PM-QPSK is employed in the EON16. However, this does not happen when employing PM-8QAM in the DT12.

\section{RESULTS}

Simulations focused on the effects of different resource partitioning strategies. Three different allocation strategies are shown in Figure 1, where the 40-30-30 profile has been offered:

- NOP (No partitioning): The connection demands are allocated without any distinction, using all the spatial channels and occupying them from low to high spatial channels using first-fit allocation.

- SPAP (Spatial partitioning): Each type of connections uses a predetermined number of spatial channels. Specifically, when 12 spatial channels are considered, $100 \mathrm{G}$ connections are allocated over the first spatial channel, $400 \mathrm{G}$ ones over spatial channels 2 to 4 , and $1 \mathrm{~Tb} / \mathrm{s}$ connections can use channels from 5 to 12 .

- SPECP (Spectral Partitioning): The number of slots allocated to each type of connection is the exact ratio corresponding to it. As 12 spatial channels are considered, a total number of $12 * 320=3840$ FS are available. Therefore, applying the respective percentages of each type of connections to this amount of spectrum, and assuming that the EON16 topology is used (values for DT12 topology were derived in previous Section 2) it results in 334 FS for $100 \mathrm{G}$ connections, 1002 FS assigned to 400G ones, while the remainder 2504 FS are reserved for $1 \mathrm{Tbit} / \mathrm{s}$ connections.

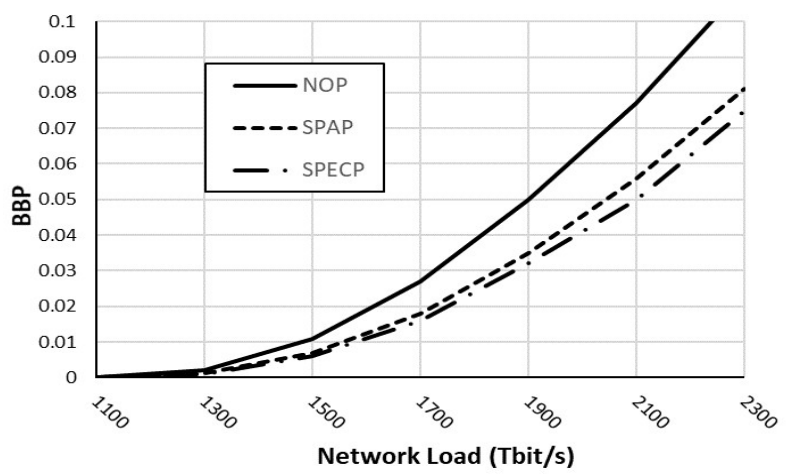

(a)

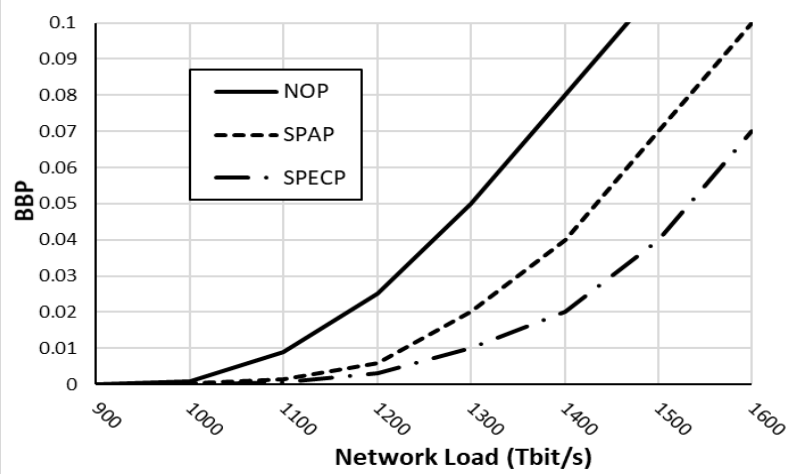

(b)

Figure 1. Bandwidth blocking probability vs. average supported network load in the DT12 (a) and EON16 (b) networks when 40-30-30 traffic profile is generated. 12 spatial channels per link are assumed.

Some conclusions can be extracted from Figure 1. First and foremost, both SPAP and SPECP are always beneficial in terms of supported load at any BBP value versus NOP, providing SPECP the best performance in general. Specifically, it is observed that gains in the EON16 network are clearly higher than those obtained in the DT12, where the performance of all options is closer. This happens as a result of the higher differences between larger and smaller connections sizes when employing PM-QPSK (EON16 network). Looking at the load values for $\mathrm{BBP}=1 \%$, these are 1490, 1560 and $1580 \mathrm{Tbit} / \mathrm{s}$ for NOP, SPAP and SPECP in DT12, respectively (Figure 1.a). In contrast, in the EON16 (Figure 1.b) the relative differences are clearly higher, namely, 1110, 1220 and $1300 \mathrm{Tbit} / \mathrm{s}$ at $\mathrm{BBP}=1 \%$. While load gains range from $4 \%$ to $6 \%$ in the DT 12 , they rise to a range from $10 \%$ to $17 \%$ in the EON16. A similar behaviour was observed for other traffic profiles and different number of spatial 
channels [10], and it is concluded that gain obtained depends on these parameters but spectral partitioning becomes always beneficial in terms of supported load at any BBP, even when there is some deviation with respect to the expected traffic profile. In addition, while the blocked connections are almost exclusively of $1 \mathrm{~Tb} / \mathrm{s}$ in the NOP case, they are fairly distributed among the different types when SPAP or SPECP are applied.

Summarizing the results obtained during simulations, SPECP is always the best case, but its advantage in front of SPAP becomes almost negligible when increasing the number of spatial channels, regardless of the considered topology. Extra network load supported by applying spectrum partition mechanisms ranges from $3 \%$ to $16 \%$ depending on the different network topologies, number of spatial channels and traffic conditions.

\section{CONCLUSIONS}

The effects of partitioning spectral resources in Flex-Grid/SDM networks have been analysed. Taking into account that the fragmentation of the spectrum limits the performance of this type of networks, the isolated allocation of the different types of connections is proposed by dividing the available resources. Two different strategies have been compared: SPECP, where the spectral resources assigned to each type of connection correspond to the expected consumed spectrum (according to the generated traffic profile); and, SPAP, where an integer number of spatial channels is assigned to each type of connection. When the number of spatial channels is high and / or the percentages of the different connection types are similar, the differences between the two strategies are reduced and the performance achieved when using SPAP is close to that of SPECP.

\section{ACKNOWLEDGEMENTS}

This work has been partially funded by Spanish projects TWINS (TEC2017-90097-R) and ALLIANCE (TEC2017-90034-C2-1-R).

\section{REFERENCES}

[1] O. Gerstel, M. Jinno, A. Lord, S. J. Ben Yoo, "Elastic optical networking: A new dawn for the optical layer?,” IEEE Commun. Mag., vol. 50, no. 2, pp. 12-20, Feb. 2012.

[2] D. J. Richardson, J. M. Fini, L. E. Nelson, "Space Division Multiplexing in Optical Fibres," Nat. Photonics, vol. 7, pp. 354-362, 2013.

[3] D. Klonidis, F. Cugini, O. Gerstel, M. Jinno, V. Lopez, E. Palkopoulou, M. Sekiya, D. Siracusa, G. Thouénon, C. Betoule, "Spectrally and spatially flexible optical network planning and operations," IEEE Commun. Mag., vol. 53, no. 2, pp. 69-78, 2015.

[4] B. C. Catterjee, N. Sarma, E. Oki, "Routing and Spectrum Allocation in Elastic Optical Networks: A Tutorial," IEEE Commun. Surv. Tut., vol. 17, no. 3, pp. 1776-1800, third quarter 2015.

[5] J. Comellas, L. Vicario, G. Junyent, "Proactive defragmentation in elastic optical networks under dynamic load conditions," Photonic Network Communications, vol. 36, pp. 26-34, 2018.

[6] Y. Qiu, "Group-based spectrum assignment in dynamic flex-grid optical networks," Optical Fiber Technology, vol. 19, no. 5, pp. 437-445, Oct. 2013.

[7] R. Wang, B. Mukherjee, "Spectrum management in heterogeneous bandwidth optical networks," Optical Switch. and Netw., vol. 11, pp. 83-91, Jan. 2014.

[8] R. Rumipamba-Zambrano, F.-J. Moreno-Muro, J. Perelló, P. Pavón-Mariño, S. Spadaro, "Space Continuity Constraint in Dynamic Flex-Grid/SDM Optical Core Networks: An Evaluation with Spatial and Spectral Super-channels," Computer Communications, vol. 126, pp. 38-49, Aug. 2018.

[9] B. Shariati, A. Mastropaolo, N.-P. Diamantopoulos, J. M. Rivas-Moscoso, D. Klonidis, I. Tomkos, "Physical-layer-aware performance evaluation of SDM networks based on SMF bundles, MCFs, and FMFs, ” J. Opt. Commun. Netw., vol. 10, no. 9, pp. 712-722, Sep. 2018.

[10] J. Comellas, J. Perelló, J. Solé-Pareta, G. Junyent, "Spatial Partitioning for Proactive Spectrum Fragmentation Avoidance in Flex-Grid/SDM Dynamic Optical Core Networks", submitted to Photonic Network Communications. 